Asian J. Med. Biol. Res. 2020, 6 (2), 283-293; doi: 10.3329/ajmbr.v6i2.48073

\author{
Asian Journal of \\ Medical and Biological Research \\ ISSN 2411-4472 (Print) 2412-5571 (Online) \\ www.ebupress.com/journal/ajmbr
}

\title{
Article \\ Status of chemicals and aqua-drugs used for freshwater fish health management at Rangpur district of Bangladesh
}

\author{
Soumen Das, Mst. Nahid Akter ${ }^{*}$ and Mst. Masuma Khatun \\ Dept. of Aquaculture, Faculty of Fisheries, Hajee Mohammad Danesh Science and Technology University, \\ Dinajpur 5200, Bangladesh
}

${ }^{*}$ Corresponding author: Mst. Nahid Akter, Dept. of Aquaculture, Faculty of Fisheries, Hajee Mohammad Danesh Science and Technology University, Dinajpur 5200, Bangladesh. Phone: +8801717526074; E-mail: mstnahidakter@gmail.com

Received: 18 May 2020/Accepted: 28 June 2020/ Published: 30 June 2020

\begin{abstract}
In order to know the existing situation of different chemicals and aqua-drugs used for fish health management an investigation was conducted following a time frame of May, 2019 to October, 2019 at Rangpur district of Bangladesh. Data were collected from the randomly selected fish farmers, company representatives and chemical sellers through questionnaire interview, personal contact and Focus Group Discussion (FGD). Farmers were facing several water quality problems associated with different fish diseases. To overcome those obstacles they use variety of chemicals inclulding lime, salt, potassium permanganate, urea, TSP, phitkiri and copper sulphate. Farmers used different chemicals for various purposes like Aquapure, Biopond, Zeofresh, JVzeolite, Zeorich, Megazeo plus, Geo-ren etc. for pond preparation and water quality management; Sumithion, Engreb, I-mec, Sanmarine etc. for eradication of unwanted species; Oxymax, Oxymore, Oxylife, Aci-ox, Oxyrich, Oxy-aqua, Oxyren etc. to increase oxygen concentration and Gasonil, Gastrap, Bioaqua-50, Gasonex, Metrix, Pondkleen etc. for reducing harmful gases in pond water. Used disinfectants like Timsen, Virex, Polgard+, , Micronil etc.; antibiotics include Renamycin, Eskamycin, Bactitab, Cotrim vet bolus, Chemycin, Aquamycin etc.; Aqua photo, Safegut, Biomax, Profs, Pond care etc. were the used probiotics where various growth promoters include Panvit aqua, Nutrigel, Aquazyme, Spa gelly, Charger gel etc. Some problems were reported regarding the use of chemicals such as their indiscriminate use, method of application, time of application and inappropriate doses.
\end{abstract}

Keywords: aquaculture; chemicals and aqua-drugs; fish health management; Rangpur

\section{Introduction}

Aquaculture is one of the fast growing food production divisions in the world. This sector has expanded, diversified, intensified and technologically advanced in Bangladesh over the last few decades (Shamsuzzaman and Biswas, 2012). The well-being of aquaculture can be maintained by good quality management practices. In aquatic animal health management, there has been an increasing trend of using aqua-medicines and chemicals due to the expansion of aquaculture in Bangladesh (Hossain et al., 2014). Farmers are using various types of compounds in fish health management and disease treatment such as growth promoters, antibiotics, disinfectants, probiotics and to develop water quality and to increase dissolved oxygen concentration (Alam and Rashid, 2014). In addition, chemicals and drugs can also play effective role in soil and water management, improvement of aquatic productivity, formulation of feed, reproduction of aquatic creatures, processing and value enhancement of the ultimate product (Subasinghe et al., 1996). In Bangladesh, approximately 400 different types of aqua-medicines are now produced and marketed by 100 pharmaceutical companies (Alam and Rashid, 2014). Presently lots of chemical industries and chemical sellers influence fish farmers to use these chemicals in their culture units, although most of the farmers have not adequate knowledge about the drug 
stability and effectivity (Karim and Stellwagen, 1998; BFRI, 1999; Faruk et al., 2004). This ignorance drives fish farmers to try several chemicals one by one based on their output. They use doses of any particular chemicals either from their own experience, from the instructions on the package of that product or by discussing with the chemical sellers or farmers. Therefore, the proper doses of these chemicals are repeatedly neglected that may eventually be a threat for aquaculture.

Considering the above facts, the present study was conducted in Rangpur district of Bangladesh with its purposes for identifying the diverse group of aqua drugs and chemicals used for aquatic health management, purposes of using, their active ingredients, recommended and applied dosages, price and sources.

\section{Materials and Methods}

\subsection{Study area and period}

To investigate the status of chemicals and aqua-drugs used for freshwater fish health management Rangpur district was selected. Data collection period was from May, 2019 to October, 2019.

\subsection{Flow chart of the research methodology}

A clear and effective routine work was done to meet the objectives of the research work. The study was undertaken and completed according to the following order of methodology:

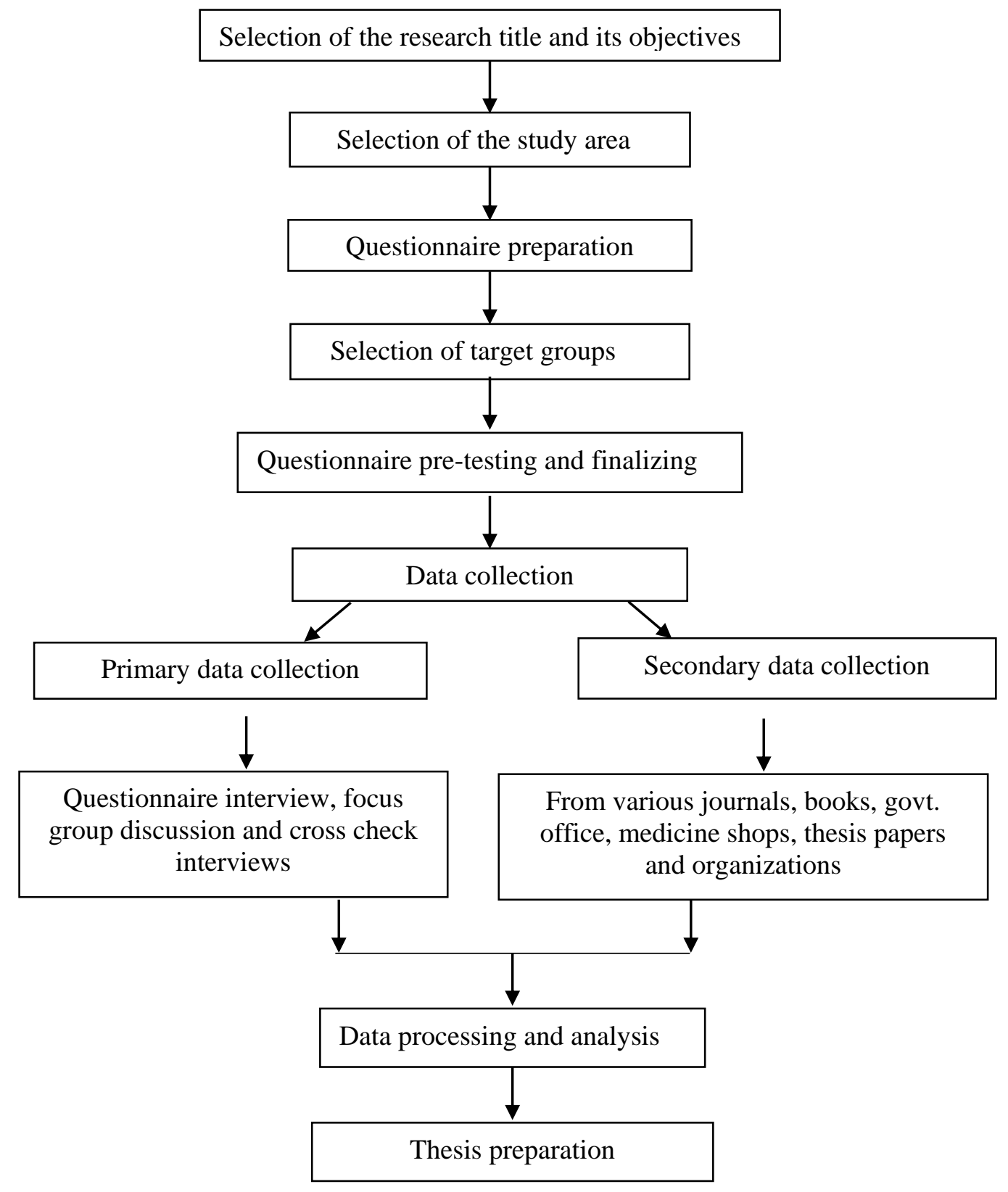


2.3. Preparation of questionnaire

Semi-structured questionnaires were made and field tested. To reach the objectives, necessary modifications were made based on the feedback. Questionnaires were prepared for data collection from the fish farmers, aquamedicine producing companies and chemical sellers.

\subsection{Target groups}

Questionnaires were prepared for investigation and to achieve information. There were 100 fish farmers, 12 technical peoples of different drug producing companies and 17 chemical or drug shops were interviewed during the study period.

\subsection{Data collection}

Data was collected through questionnaire interview, personal contact, market surveys and Participatory Rural Appraisal (PRA) tool like Focus Group Discussion (FGD) with fish farmers, farm workers, medicine sellers and representative of different aqua-medicine producing companies in the selected area. Both the primary and secondary sources were utilized to collect data. Primary data were collected through simple interview with the respondents while carrying the survey. Secondary data helped to identify the problems and to confirm the primary data.

\subsection{Analysis of data}

The data were coded and inputted in a computer for further analysis. Data sheets were matched with questionnaires to confirm the exactness of data entered. MS Word and MS Excel have been used for processing and analysis, and presented in textual and tabular forms to meet the objectives.

\section{Results}

\subsection{Water quality problems faced by the fish farmers}

In the study area, the fish farmers reported various types of water quality problems in fish pond during culture period. These includes dissolve oxygen (DO) deficiency (95\% of farmers), high ammonia (74\%), algal bloom $(37 \%)$, turbidity (28\%), $\mathrm{pH}(19 \%)$ and poor phytoplankton (14\%) (Table 1).

\subsection{Diseases faced by the fish farmers}

In the study area, various types of fish diseases were occurred mainly during the winter season. Fishes were affected by different types of fish diseases such as EUS (69.32\% of farmers), tail and fin rot (59.09\%), argulus (55.68\%), gill rot (43.18\%), dropsy (42.05\%), exophthalmia (13.64\%), CCVD (5.68\%) (Table 2).

\subsection{Some commonly used traditional chemicals}

From the present study, it was found that Lime, Potassium permanganate, Salt, Phitkari, Copper sulphate, Urea and TSP were commonly used by the selected farmers. The list of those used chemicals, their applied doses, price and sources are shown in (Table 3).

\subsection{Chemicals used to remove unwanted species}

For the removal of unwanted species, the farmers used Engreb, Nigotox aqua, Rota plus, Sanmarine, Sumithion, Tea seed meal and I-mec. Different information regarding to those chemicals are given in (Table 4).

\subsection{Chemicals used for pond preparation and water quality management}

The fish farmers of the study area used some chemicals for pond preparation and water quality management such as Aquapure, Biopond, Zeofresh, JV-zeolite, Zeorich, and others are shown in Table 5.

\subsection{Chemicals used to increase oxygen concentration}

In the investigation area, some chemicals were used by fish farmers to increase oxygen concentration in their pond. These chemicals are listed below (Table 6) with their trade name, active ingredients, recommended dose, applied dose, price and source/manufacturer.

\subsection{Aqua-medicines used to remove harmful gasses in the study area}

Farmers of the Rangpur region used some aqua-medicines to remove harmful gasses from their culture unit. The trade name, active ingredients, recommended dose, applied dose, price and source/manufacturer of those aquamedicines are given in Table 7. 
3.8. Disinfectants used in the study area

The fish farmers of the study area used some disinfectants to keep their pond free from pathogens. These aquamedicines are listed below with their trade name, active ingredients, recommended dose, applied dose, price and source/manufacturer (Table 8).

\subsection{Antibiotics used against diseases in the study area}

From the study, it was observed that antibiotics were used by the farmers against fish diseases at Rangpur district. The trade name, active ingredients, recommended dose, applied dose, price and source/manufacturer of those antibiotics are given in (Table 9).

\subsection{Probiotics used in the study area}

The selected fish farmers were also used various types of probiotics. The trade name, active ingredients, recommended dose, applied dose, price and source/manufacturer of some probiotics are shown in (Table 10).

\subsection{Growth promoters used in the study area}

Several growth promoters were used by the fish farmers of the Rangpur district. These growth promoters are given below (Table 11) with their trade name, active ingredients, recommended dose, applied dose, price and source/manufacturer.

\subsection{Knowledge of aqua chemicals and drugs}

In the study area, most of the farmers (76\%) were known of the aqua-medicines from company representatives through the seminars arranged by the company personnel, while (58\%) of the farmers acquired knowledge on aqua-medicines from discussion with chemical sellers, hatchery owners and other farmers. About $47 \%$ farmers obtain knowledge from government organizations (GOs) (Table 12).

\subsection{Problems faced by the fish farmers in the study area}

Several problems were faced by the fish farmers in the study area including diseases ( $88 \%$ of farmers), low quality of fish seed (63\%), lack of technical knowledge (58\%), lack of finance (55\%), low price of the end product $(46 \%)$, insufficient water in dry season (41\%), price of feeds and chemicals $(39 \%)$, flood/overflow $(36 \%)$, lack of manpower (30\%), problem of pond leasing (27\%), theft of fish (21\%) (Table 13).

Table 1. Water quality problems faced by the fish farmers in the study area.

\begin{tabular}{|c|c|c|c|c|c|}
\hline $\begin{array}{l}\text { Water quality } \\
\text { problems }\end{array}$ & $\begin{array}{l}\text { Prevalence } \\
(\%)\end{array}$ & Death (\%) & Treatment & $\begin{array}{l}\text { Number of farmers } \\
(\mathrm{n}=\mathbf{1 0 0})\end{array}$ & $\begin{array}{l}\% \text { of } \\
\text { farmers }\end{array}$ \\
\hline DO deficiency & $40-60$ & $2-5$ & $\begin{array}{l}\text { Exchange of water, sodium } \\
\text { per-carbonate }\end{array}$ & 95 & 95 \\
\hline Ammonia & $20-40$ & $2-10$ & $\begin{array}{l}\text { Exchange of water, Үисca } \\
\text { schidigera extract }\end{array}$ & 74 & 74 \\
\hline Algal bloom & $30-50$ & $0-1$ & $\begin{array}{l}\text { Withdrawal of bloom using } \\
\text { straw rope, probiotics }\end{array}$ & 37 & 37 \\
\hline Turbidity & $30-50$ & $0-2$ & Lime, zeolite & 28 & 28 \\
\hline $\mathrm{pH}$ & $20-40$ & $4-10$ & $\begin{array}{l}\text { Exchange of water, lime } \\
\text { (when low), Yucca schidigera } \\
\text { extract (when high) }\end{array}$ & 19 & 19 \\
\hline Poor phytoplankton & $20-30$ & $0-1$ & Urea, TSP, zeolite & 14 & 14 \\
\hline
\end{tabular}


Asian J. Med. Biol. Res. 2020, 6 (2)

Table 2. Diseases faced by the fish farmers in the study area.

\begin{tabular}{|c|c|c|c|c|c|}
\hline Disease & Clinical signs & $\begin{array}{l}\text { Prevalence } \\
(\%)\end{array}$ & Death $(\%)$ & $\begin{array}{l}\text { Number of } \\
\text { farmers }(n=88)\end{array}$ & $\begin{array}{l}\% \text { of } \\
\text { farmers }\end{array}$ \\
\hline EUS & Red spot and infection & $30-50$ & $5-20$ & 61 & 69.32 \\
\hline Tail and fin rot & Reddish color, broken tail and fin & $20-50$ & $2-20$ & 52 & 59.09 \\
\hline Argulus & $\begin{array}{l}\text { Rubbing or flashing against solid, excess } \\
\text { mucous }\end{array}$ & $20-40$ & $2-10$ & 49 & 55.68 \\
\hline Gill rot & Gill swelled and discolored gradually & $20-40$ & $5-20$ & 38 & 43.18 \\
\hline Dropsy & Swollen abdomen & $10-30$ & $2-15$ & 37 & 42.05 \\
\hline Exophthalmia & Eye swollen & $10-25$ & $2-15$ & 12 & 13.64 \\
\hline CCVD & $\begin{array}{l}\text { Lesion on mouth, white spot in head and } \\
\text { body, infected fish remain flat on surface }\end{array}$ & 70-95 & $80-90$ & 5 & 5.68 \\
\hline
\end{tabular}

Table 3. List of commonly used chemicals.

\begin{tabular}{|c|c|c|c|}
\hline Trade name & Applied dose & Price (TK.) & Source \\
\hline Lime & $\begin{array}{l}\text { Pond preparation: } 1 \mathrm{~kg} / \mathrm{dec} \text { (sandy bottom), } 1-2 \mathrm{~kg} / \mathrm{dec} \\
\text { (clay bottom) } \\
\text { During culture: } 200-250 \mathrm{~g} / \mathrm{dec}\end{array}$ & $20-22 / \mathrm{kg}$ & Chemical seller \\
\hline $\begin{array}{l}\text { Potassium permanganate } \\
\left(\mathrm{KMnO}_{4}\right)\end{array}$ & $0.5-2 \mathrm{~g} / \mathrm{dec}$ & $20 / 10 \mathrm{~g}$ & Chemical seller \\
\hline Salt $(\mathrm{NaCl})$ & $100-200 \mathrm{~g} / \mathrm{dec}$ & $18 / \mathrm{kg}$ & Chemical seller \\
\hline Phitkari & $8-10 \mathrm{~g} / \mathrm{dec}$ & $60-80 / \mathrm{kg}$ & Chemical seller \\
\hline $\begin{array}{l}\text { Copper sulphate } \\
{\left[\mathrm{CuSO}_{4} \cdot 5 \mathrm{H}_{2} \mathrm{O}\right]}\end{array}$ & $5-10 \mathrm{~g} / \mathrm{dec}$ & $200-220 / \mathrm{kg}$ & Chemical seller \\
\hline Urea & $\begin{array}{l}200-300 \mathrm{~g} / \mathrm{dec} \text { (pond preparation), 100-150 g/dec (during } \\
\text { culture) }\end{array}$ & $16-17 / \mathrm{kg}$ & Chemical seller \\
\hline TSP & $\begin{array}{l}100-200 \mathrm{~g} / \mathrm{dec} \text { (pond preparation), } \\
50-100 \mathrm{~g} / \mathrm{dec} \text { (during culture) }\end{array}$ & $22 / \mathrm{kg}$ & Chemical seller \\
\hline
\end{tabular}

Table 4. List of chemicals used to remove unwanted species.

\begin{tabular}{|c|c|c|c|c|c|}
\hline Trade name & Active ingredients & $\begin{array}{l}\text { Recommended } \\
\text { dose }\end{array}$ & Applied dose & Price (TK.) & $\begin{array}{l}\text { Source/ } \\
\text { Manufacturer }\end{array}$ \\
\hline Engreb & Cypermethrine $10 \%$ & $7 \mathrm{ml} / \mathrm{dec}$ & $5-8 \mathrm{ml} / \mathrm{dec}$ & $125 / 100 \mathrm{ml}$ & $\begin{array}{l}\text { Eon Animal Health } \\
\text { Ltd. }\end{array}$ \\
\hline Nigotox aqua & Trichlorphon $40 \%$ & $12-13 \mathrm{ml} / \mathrm{dec}$ & $10-12 \mathrm{ml} / \mathrm{dec}$ & $240 / 250 \mathrm{ml}$ & $\begin{array}{l}\text { Chemist } \\
\text { Laboratories Ltd. }\end{array}$ \\
\hline Rota plus & Rotenone 9\% & $\begin{array}{l}20-30 \mathrm{~g} / \mathrm{dec} / \mathrm{ft} . \\
\text { water }\end{array}$ & $\begin{array}{l}20-25 \mathrm{~g} / \mathrm{dec} / \mathrm{ft} . \\
\text { water }\end{array}$ & $480 / \mathrm{kg}$ & $\begin{array}{l}\text { ACI Animal Health } \\
\text { Ltd. }\end{array}$ \\
\hline Sanmarine & $\begin{array}{l}\text { Cypermethrine } 10 \\
\text { E.C. }\end{array}$ & $5-7 \mathrm{ml} / \mathrm{dec}$ & $5-7 \mathrm{ml} / \mathrm{dec}$ & $390 / 400 \mathrm{ml}$ & Macdonald BD. \\
\hline Sumithion & Fanitrothion & $4-5 \mathrm{ml} / \mathrm{dec}$ & $4-6 \mathrm{ml} / \mathrm{dec}$ & $162 / 100 \mathrm{ml}$ & Chemical seller \\
\hline Tea seed meal & Seponin $15-16 \%$ & $\begin{array}{l}0.8-1 \mathrm{~kg} / \mathrm{dec} / 5 \mathrm{ft} \text {. } \\
\text { water }\end{array}$ & $1 \mathrm{~kg} / \mathrm{dec}$ & $4500 / 50 \mathrm{~kg}$ & $\begin{array}{l}\text { ACI Animal Health } \\
\text { Ltd. }\end{array}$ \\
\hline I-mec & Ivermectine & $\begin{array}{l}6 \mathrm{ml} / \mathrm{dec} \\
\text { (3ft. water) }\end{array}$ & $5-6 \mathrm{ml} / \mathrm{dec}$ & $265 / 100 \mathrm{ml}$ & $\begin{array}{l}\text { Eon Animal Health } \\
\text { Ltd. }\end{array}$ \\
\hline
\end{tabular}


Asian J. Med. Biol. Res. 2020, 6 (2)

Table 5. List of chemicals used for pond preparation and water quality management.

\begin{tabular}{|c|c|c|c|c|c|}
\hline $\begin{array}{l}\text { Trade } \\
\text { name }\end{array}$ & Active ingredients & $\begin{array}{l}\text { Recommended } \\
\text { dose }\end{array}$ & Applied dose & Price (TK.) & $\begin{array}{l}\text { Source/ } \\
\text { Manufacturer }\end{array}$ \\
\hline JV zeolite & Natural geolite & $6-8 \mathrm{~kg} / 33 \mathrm{dec}$ & $\begin{array}{l}6.5-7 \mathrm{~kg} / 33 \mathrm{dec} \\
(200 \mathrm{~g} / \mathrm{dec})\end{array}$ & $565 / 10 \mathrm{~kg}$ & $\begin{array}{l}\text { Eon Animal Health } \\
\text { Ltd. }\end{array}$ \\
\hline Geo-prime & $\begin{array}{l}\text { Natural green geolite } \\
100 \%\end{array}$ & $200-250 \mathrm{~g} / \mathrm{dec}$ & $200 \mathrm{~g} / \mathrm{dec}$ & $500 / 10 \mathrm{~kg}$ & $\begin{array}{l}\text { SK+F Pharmaceuticals } \\
\text { Ltd. }\end{array}$ \\
\hline Zeorich & Natural geolite $100 \%$ & $100-200 \mathrm{~g} / \mathrm{dec}$ & $100 \mathrm{~g} / \mathrm{dec}$ & $535 / 10 \mathrm{~kg}$ & Opsonin Agrovet \\
\hline $\begin{array}{l}\text { Megazeo } \\
\text { plus }\end{array}$ & $\begin{array}{l}\mathrm{SiO}_{2}, \mathrm{Al}_{2} \mathrm{O}_{3}, \mathrm{Fe}_{2} \mathrm{O}_{3}, \\
\mathrm{CaO}, \mathrm{MgO}, \mathrm{Na}_{2} \mathrm{O}, \mathrm{K}_{2} \mathrm{O} \\
\text { and } \mathrm{Mn}\end{array}$ & $200 \mathrm{~g} / \mathrm{dec} /$ month & $200-250 \mathrm{~g} / \mathrm{dec}$ & $400 / 10 \mathrm{~kg}$ & $\begin{array}{l}\text { ACI Animal Health } \\
\text { Ltd. }\end{array}$ \\
\hline Aqua pure & $\begin{array}{l}\text { Natural sodium } \\
\text { alluminium silicate }\end{array}$ & $10-16 \mathrm{~kg} / \mathrm{acre}$ & $\begin{array}{l}10 \mathrm{~kg} / \mathrm{acre} \\
(100 \mathrm{~g} / \mathrm{dec})\end{array}$ & $580 / 5 \mathrm{~kg}$ & $\begin{array}{l}\text { Square } \\
\text { Pharmaceuticals Ltd. }\end{array}$ \\
\hline Biopond & Geolite and probiotic & $2-3 \mathrm{~kg} / \mathrm{acre} / 15$ days & $\begin{array}{l}2-2.5 \mathrm{~kg} / \mathrm{acre} \\
(20-25 \mathrm{~g} / \mathrm{dec})\end{array}$ & $1035 / 2 \mathrm{~kg}$ & $\begin{array}{l}\text { SK+F Pharmaceuticals } \\
\text { Ltd. }\end{array}$ \\
\hline Zeolite gold & $\mathrm{SiO}_{3}, \mathrm{MgO}, \mathrm{CaOetc}$ & $\begin{array}{l}200-250 \mathrm{~g} / \mathrm{dec} / \\
\text { month }\end{array}$ & $200 \mathrm{~g} / \mathrm{dec}$ & $530 / 10 \mathrm{~kg}$ & Fish Tech BD Ltd. \\
\hline Zeo fresh & $\begin{array}{l}\mathrm{SiO}_{2}, \mathrm{Al}_{2} \mathrm{O}_{3}, \mathrm{Fe}_{2} \mathrm{O}_{3} \\
\mathrm{CaO}, \mathrm{MgO}, \mathrm{Na}_{2} \mathrm{O}, \\
\mathrm{K}_{2} \mathrm{O} \text { and } \mathrm{Mn}\end{array}$ & $10 \mathrm{~kg} / \mathrm{acre}$ & $\begin{array}{l}10 \mathrm{~kg} / \mathrm{acre} \\
(100 \mathrm{~g} / \mathrm{dec})\end{array}$ & $420 / 10 \mathrm{~kg}$ & $\begin{array}{l}\text { Square } \\
\text { Pharmaceuticals Ltd. }\end{array}$ \\
\hline Geo-ren & $\begin{array}{l}\text { Aluminum sodium } \\
\text { silicate }\end{array}$ & $20-25 \mathrm{~kg} / \mathrm{acre}$ & $\begin{array}{l}20 \mathrm{~kg} / \mathrm{acre} \\
(200 \mathrm{~g} / \mathrm{dec})\end{array}$ & $650 / 10 \mathrm{~kg}$ & Renata Animal Health \\
\hline $\begin{array}{l}\text { Acme } \\
\text { zeolite }\end{array}$ & $\begin{array}{l}\text { Aluminum sodium } \\
\text { silicate }\end{array}$ & $20-30 \mathrm{~kg} / \mathrm{acre}$ & $\begin{array}{l}20 \mathrm{~kg} / \mathrm{acre} \\
(200 \mathrm{~g} / \mathrm{dec})\end{array}$ & $280 / 10 \mathrm{~kg}$ & $\begin{array}{l}\text { ACME } \\
\text { Laboratories }\end{array}$ \\
\hline
\end{tabular}

Table 6. List of chemicals used to increase oxygen concentration.

\begin{tabular}{|c|c|c|c|c|c|}
\hline $\begin{array}{l}\text { Trade } \\
\text { name }\end{array}$ & Active ingredients & Recommended dose & Applied dose & $\begin{array}{l}\text { Price } \\
\text { (TK.) }\end{array}$ & $\begin{array}{l}\text { Source/ } \\
\text { Manufacturer }\end{array}$ \\
\hline Aci-Ox & $\begin{array}{l}\text { Sodium per carbonate } \\
2 \mathrm{Na}_{2} \mathrm{CO}_{3} .3 \mathrm{H}_{2} \mathrm{O}_{2}\end{array}$ & $\begin{array}{l}\text { Normal: } \\
\text { 3-4 g/dec } \\
\text { Extreme: } \\
5-7 \mathrm{~g} / \mathrm{dec}\end{array}$ & $7-8 \mathrm{~g} / \mathrm{dec}$ & $510 / \mathrm{kg}$ & $\begin{array}{l}\text { ACI Animal Health } \\
\text { Ltd. }\end{array}$ \\
\hline Oxy-A & Sodium percarbonate & $3-4 \mathrm{~g} / \mathrm{dec}$ & $4-5 \mathrm{~g} / \mathrm{dec}$ & $550 / \mathrm{kg}$ & $\begin{array}{l}\text { ACME Laboratories } \\
\text { Ltd. }\end{array}$ \\
\hline Oxy-aqua & Sodium percarbonate & $2.5-5 \mathrm{~g} / \mathrm{dec}$ & $4-5 \mathrm{~g} / \mathrm{dec}$ & $690 / \mathrm{kg}$ & $\begin{array}{l}\text { Navana Animal } \\
\text { Health }\end{array}$ \\
\hline Oxyflox & Sodium percarbonate & $\begin{array}{l}\text { Normal: } \\
\text { 3-4 g/ dec } \\
\text { Extreme: } \\
5-6 \text { g/dec }\end{array}$ & $5-6 \mathrm{~g} / \mathrm{dec}$ & $500 / \mathrm{kg}$ & $\begin{array}{l}\text { Chemist Laboratories } \\
\text { Ltd. }\end{array}$ \\
\hline Oxy gold & Sodium percarbonate & $2.5-5 \mathrm{~g} / \mathrm{dec}$ & $4-6 \mathrm{~g} / \mathrm{dec}$ & $635 / \mathrm{kg}$ & Fish Tech Ltd. \\
\hline $\begin{array}{l}\text { Oxyrich } \\
\text { Tab }\end{array}$ & $\begin{array}{l}\text { Sustained release } \mathrm{O}_{2} \\
12 \%\end{array}$ & $5-12 \mathrm{tab} / \mathrm{dec}$ & $5-10 \mathrm{tab} / \mathrm{dec}$ & $650 / \mathrm{kg}$ & $\begin{array}{l}\text { Opsonin } \\
\text { Agrovet }\end{array}$ \\
\hline Oxymax & Sodium percarbonate & $\begin{array}{l}\text { Normal: } \\
2.5-3 \mathrm{~g} / \mathrm{dec} \\
\text { Extreme: } \\
5-10 \mathrm{~g} / \mathrm{dec}\end{array}$ & $4-10 \mathrm{~g} / \mathrm{dec}$ & $700 / \mathrm{kg}$ & Eon Animal Health \\
\hline Oxylife & $\begin{array}{l}\text { Oxygen } \\
\text { Probiotics }\end{array}$ & $4-5 \mathrm{~g} / \mathrm{dec}$ & $4-6 \mathrm{~g} / \mathrm{dec}$ & $650 / \mathrm{kg}$ & $\begin{array}{l}\text { Square } \\
\text { Pharmaceuticals Ltd. }\end{array}$ \\
\hline Oxymore & $\begin{array}{l}\text { Sodium carbonate per- } \\
\text { oxihydrate } 90 \%\end{array}$ & $\begin{array}{l}\text { Normal: } \\
2-5 \mathrm{~g} / \mathrm{dec} \\
\text { Extreme: } \\
7-10 \mathrm{~g} / \mathrm{dec}\end{array}$ & $4-10 \mathrm{~g} / \mathrm{dec}$ & $740 / \mathrm{kg}$ & $\begin{array}{l}\text { SK+F } \\
\text { Pharmaceuticals Ltd. }\end{array}$ \\
\hline Oxyren & Sodium percarbonate & $2-3 \mathrm{~g} / \mathrm{dec}$ & $4-5 \mathrm{~g} / \mathrm{dec}$ & $650 / \mathrm{kg}$ & $\begin{array}{l}\text { Renata } \\
\text { Pharmaceuticals Ltd. }\end{array}$ \\
\hline
\end{tabular}


Table 7. List of aqua-medicine used to remove harmful gasses in the study area.

\begin{tabular}{|c|c|c|c|c|c|}
\hline Trade name & Active ingredients & $\begin{array}{l}\text { Recommended } \\
\text { dose }\end{array}$ & Applied dose & Price (TK.) & $\begin{array}{l}\text { Source/ } \\
\text { Manufacturer }\end{array}$ \\
\hline Bio-Aqua-50 & $\begin{array}{l}\text { Yucca plant extract, } \\
\text { Saponin components }\end{array}$ & $3-4 \mathrm{ml} / \mathrm{dec}$ & $3-4 \mathrm{ml} / \mathrm{dec}$ & 298/100ml & $\begin{array}{l}\text { Eon Animal Health } \\
\text { Products Ltd. }\end{array}$ \\
\hline Gasonex (+) & $\begin{array}{l}\text { Pseudomonas sp., } \\
\text { Bacillus subtilis, } \\
\text { Nitrococcus } \mathrm{sp} .\end{array}$ & $200-400 \mathrm{~g} / \mathrm{acre}$ & $\begin{array}{l}300-400 \\
\text { g/acre } \\
(3-4 \mathrm{~g} / \mathrm{dec})\end{array}$ & $445 / 100 \mathrm{~g}$ & Fishtech BD Ltd. \\
\hline Gastrap & $\begin{array}{l}\text { Enzyme and } \\
\text { probiotics }\end{array}$ & $\begin{array}{l}200 \mathrm{~g} / \mathrm{acre} / 3-6 \text { feet } \\
\text { water }\end{array}$ & $\begin{array}{l}200-400 \\
\text { g/acre } \\
(2-4 \mathrm{~g} / \mathrm{dec})\end{array}$ & $326 / 100 \mathrm{~g}$ & $\begin{array}{l}\text { Square } \\
\text { Pharmaceuticals Ltd. }\end{array}$ \\
\hline Yucca gold & $\begin{array}{l}\text { Yucca schidigera } \\
\text { extract }\end{array}$ & $100 \mathrm{ml} / 33 \mathrm{dec}$ & $\begin{array}{l}100-132 \\
\mathrm{ml} / 33 \mathrm{dec} \\
(3-4 \mathrm{ml} / \mathrm{dec})\end{array}$ & $1850 / 500 \mathrm{ml}$ & $\begin{array}{l}\text { ACI Animal Health } \\
\text { Ltd. }\end{array}$ \\
\hline Gasonil & $\begin{array}{l}\text { Yucca plant extract, } \\
\text { Saponin cmponents, } \\
\text { Glyco components }\end{array}$ & $150-200 \mathrm{~g} / \mathrm{acre}$ & $\begin{array}{l}300-400 \\
\text { g/acre } \\
(3-4 \mathrm{~g} / \mathrm{dec})\end{array}$ & $495 / 250 \mathrm{~g}$ & $\begin{array}{l}\mathrm{SK}+\mathrm{F} \\
\text { Pharmaceuticals Ltd. }\end{array}$ \\
\hline Metrix & $\begin{array}{l}\mathrm{Al}_{2} \mathrm{O}_{3}, \mathrm{CaO}, \mathrm{SiO}_{2}, \\
\text { Feroso ferric oxide }\end{array}$ & $6-10 \mathrm{~kg} / \mathrm{acre}$ & $\begin{array}{l}5-6 \mathrm{~kg} / \mathrm{acre} \\
(50-60 \mathrm{~g} / \mathrm{dec})\end{array}$ & $767 / 5 \mathrm{~kg}$ & $\begin{array}{l}\text { Eon Pharmaceuticals } \\
\text { Ltd. }\end{array}$ \\
\hline Pondkleen & $\begin{array}{l}\text { Yucca schidigera } \\
\text { plant extract }\end{array}$ & $100 \mathrm{ml} / 33 \mathrm{dec}$ & $\begin{array}{l}100-132 \\
\mathrm{ml} / 33 \mathrm{dec} \\
(3-4 \mathrm{ml} / \mathrm{dec})\end{array}$ & $1500 / 500 \mathrm{ml}$ & $\begin{array}{l}\text { ACI Animal Health } \\
\text { Ltd. }\end{array}$ \\
\hline Ammo check & $\begin{array}{l}\text { Extract of Yucca } \\
\text { schidigera }\end{array}$ & $\begin{array}{l}3-4 \mathrm{ml} / \mathrm{dec} / 3-5 \mathrm{ft} . \\
\text { water }\end{array}$ & $3-6 \mathrm{ml} / \mathrm{dec}$ & $250 / 100 \mathrm{ml}$ & $\begin{array}{l}\text { Navana Animal } \\
\text { Health }\end{array}$ \\
\hline Yuka & $\begin{array}{l}\text { Yucca schidigera } \\
\text { extract }\end{array}$ & $\begin{array}{l}2-3 \mathrm{ml} / \mathrm{dec} / 3-4 \mathrm{ft} . \\
\text { water }\end{array}$ & $2-5 \mathrm{ml} / \mathrm{dec}$ & $315 / 100 \mathrm{ml}$ & $\begin{array}{l}\text { Opsonin } \\
\text { Agrovet }\end{array}$ \\
\hline
\end{tabular}

Table 8. List of chemicals or aqua-drugs used as disinfectants.

\begin{tabular}{|c|c|c|c|c|c|}
\hline $\begin{array}{l}\text { Trade } \\
\text { name }\end{array}$ & Active ingredients & $\begin{array}{l}\text { Recommended } \\
\text { dose }\end{array}$ & Applied dose & Price (TK.) & $\begin{array}{l}\text { Source/ } \\
\text { Manufacturer }\end{array}$ \\
\hline Timsen & $\begin{array}{l}\mathrm{N} \text {-alkyl dimethyl benzyl } \\
\text { ammonium chloride- } 40 \%\end{array}$ & $\begin{array}{l}20 \mathrm{~g} / 33 \mathrm{dec} \quad \text { (for } \\
\text { prevention) } \\
\text { 80g/33dec. } \\
\text { (for treatment) }\end{array}$ & $1-2 \mathrm{~g} / \mathrm{dec}$ & $111 / 20 \mathrm{~g}$ & $\begin{array}{l}\text { Eon Animal } \\
\text { Health Products } \\
\text { Ltd. }\end{array}$ \\
\hline Aquakleen & $\begin{array}{l}\text { Tetra-desail Tri-methyl } \\
\text { Ammonium bromide, } \\
\text { BKC, Amaino nitrate }\end{array}$ & $0.5-1$ liter/acre & o.8-1 liter/acre & 460/1 liter & $\begin{array}{l}\text { Square } \\
\text { Pharmaceuticals } \\
\text { Ltd. }\end{array}$ \\
\hline Pond safe & $\begin{array}{l}\text { Alkyl-dimethyl benzyl- } \\
\text { ammonium } \\
\text { Chloride Solution } 80 \% \\
\text { Inert Ingredients } 20 \%\end{array}$ & $600-800 \mathrm{ml} /$ acre & $6-7 \mathrm{ml} / \mathrm{dec}$ & $1035 / 500 \mathrm{ml}$ & Fish Tech BD Ltd. \\
\hline Virex & $\begin{array}{l}\text { Potassium per-oxi } \\
\text { monosulphate } 50 \%\end{array}$ & $100 \mathrm{~g} / 33 \mathrm{dec}$ & $\begin{array}{l}100-132 \mathrm{~g} / 33 \mathrm{dec} \\
(3-4 \mathrm{~g} / \mathrm{dec})\end{array}$ & $170 / 100 \mathrm{~g}$ & $\begin{array}{l}\text { ACI Animal } \\
\text { Health Ltd. }\end{array}$ \\
\hline Polgard plus & $\begin{array}{l}3 \text {-methyl and } 4 \text { methyl } \\
\text { two chain brominated } \\
\text { compound }\end{array}$ & $500 \mathrm{ml} /$ acre & $\begin{array}{l}500-600 \mathrm{ml} / \mathrm{acre} \\
(5-6 \mathrm{ml} / \mathrm{dec})\end{array}$ & $460 / 250 \mathrm{ml}$ & Fishtech Ltd. \\
\hline $\begin{array}{l}\text { Bleaching } \\
\text { powder }\end{array}$ & Calcium hypochlorite & $50 \mathrm{~g} / \mathrm{dec}$ & $50 \mathrm{~g} / \mathrm{dec}$ & $90-100 / \mathrm{kg}$ & Chemical seller \\
\hline $\begin{array}{l}\text { Biokleen } \\
\text { aqua }\end{array}$ & $\begin{array}{l}\text { n-alkyl dimethyl benzyl } \\
\text { ammonium chloride } \\
40 \%+\text { stabilized urea } \\
60 \%\end{array}$ & $1-2 \mathrm{~g} / \mathrm{dec}$ & $1-2 \mathrm{~g} / \mathrm{dec}$ & $180 / 30 \mathrm{~g}$ & $\begin{array}{l}\text { Chemist } \\
\text { Pharmaceuticals } \\
\text { Ltd. }\end{array}$ \\
\hline Sansure & $\begin{array}{l}\text { Benzylchonium chloride } \\
80 \%\end{array}$ & $150-200 \mathrm{ml} / 33 \mathrm{dec}$ & $\begin{array}{l}165-198 \mathrm{ml} / 33 \mathrm{dec} \\
(5-6 \mathrm{ml} / \mathrm{dec})\end{array}$ & $285 / 100 \mathrm{ml}$ & $\begin{array}{l}\text { Opsonin } \\
\text { Agrovet }\end{array}$ \\
\hline Micronil & $\begin{array}{l}\text { Benzylchonium chloride } \\
80 \%\end{array}$ & 1-2 liter/acre & $\begin{array}{l}1-1.2 \text { liter/acre } \\
(10-12 \mathrm{ml} / \mathrm{dec})\end{array}$ & $300 / 100 \mathrm{ml}$ & $\begin{array}{l}\text { SK+F } \\
\text { Pharmaceuticals } \\
\text { Ltd. }\end{array}$ \\
\hline
\end{tabular}


Asian J. Med. Biol. Res. 2020, 6 (2)

Table 9. List of antibiotics used against diseases.

\begin{tabular}{|c|c|c|c|c|c|}
\hline Trade name & Active ingredients & $\begin{array}{l}\text { Recommended } \\
\text { dose }\end{array}$ & Applied dose & Price (TK.) & $\begin{array}{l}\text { Source/ } \\
\text { Manufacturer }\end{array}$ \\
\hline Renamycin & Oxytetracycline & $\begin{array}{l}2-4 \mathrm{~g} / 4-5 \mathrm{~kg} \text { feed, } \\
\text { 10days }\end{array}$ & $\begin{array}{l}1-2 \quad \mathrm{~g} / \mathrm{kg} \\
\text { feed/week }\end{array}$ & $80 / 100 \mathrm{~g}$ & $\begin{array}{l}\text { Renata } \\
\text { Pharmaceuticals Ltd }\end{array}$ \\
\hline Bactitab & Oxytetracycline $20 \%$ & $1-2 \mathrm{~g} / \mathrm{kg}$ feed/week & $\begin{array}{l}1-5 \mathrm{~g} / \mathrm{kg} \text { feed/ } \\
\text { week }\end{array}$ & $82 / 100 \mathrm{~g}$ & $\begin{array}{l}\text { ACI Animal Health } \\
\text { Ltd. }\end{array}$ \\
\hline Eskamycin & Oxytetracycline $50 \%$ & $1-2 \mathrm{~g} / \mathrm{kg}$ feed & $1-4 \mathrm{~g} / \mathrm{kg}$ feed & $180 / 100 \mathrm{~g}$ & $\mathrm{SK}+\mathrm{F}$ \\
\hline Chemycin & Oxytetracline $\mathrm{HCl} \mathrm{BP}$ & $\begin{array}{l}0.3-1 \mathrm{~g} / \mathrm{kg} \text { feed, } 5- \\
7 \text { days }\end{array}$ & $1-2 \mathrm{~g} / \mathrm{kg}$ feed & $700 / \mathrm{kg}$ & $\begin{array}{l}\text { Pharmaceuticals Ltd. } \\
\text { Chemist Laboratories } \\
\text { Ltd. }\end{array}$ \\
\hline Aquamycin & Chlortetracycline & $\begin{array}{l}1-2 \mathrm{~g} / \mathrm{kg} \text { feed, } 5 \\
\text { days' interval }\end{array}$ & $1-3 \mathrm{~g} / \mathrm{kg}$ feed & $700-800 / \mathrm{kg}$ & Fishtech BD. Ltd. \\
\hline Oxy-D Vet & $\begin{array}{l}\text { Oxytetracycline } 20 \%+ \\
\text { Doxycycline } 10 \%\end{array}$ & $1 \mathrm{~g} / \mathrm{kg}$ feed daily & $1-2 \mathrm{~g} / \mathrm{kg}$ feed & $175 / 100 \mathrm{~g}$ & $\begin{array}{l}\text { Eon Animal Health } \\
\text { Products Ltd. }\end{array}$ \\
\hline $\begin{array}{l}\text { Cotrim vet } \\
\text { bolus }\end{array}$ & $\begin{array}{l}\text { Sulphamethoxa-zole + } \\
\text { Trimethoprim }\end{array}$ & $1-2 \mathrm{~g} / \mathrm{kg}$ feed/week & $1-4 \mathrm{~g} / \mathrm{kg}$ feed & $89 / 20$ bolus & $\begin{array}{l}\text { Square } \\
\text { Pharmaceuticals Ltd. }\end{array}$ \\
\hline Oxin WS & Oxytetracycline $20 \%$ & $1-2 \mathrm{~g} / \mathrm{kg}$ feed & $1-2 \mathrm{~g} / \mathrm{kg}$ feed & $745 / \mathrm{kg}$ & $\begin{array}{l}\text { Navana Animal } \\
\text { Health }\end{array}$ \\
\hline $\begin{array}{l}\text { Otetra vet } \\
\text { power } 50\end{array}$ & Oxytetracycline & $2-3 \mathrm{~g} / \mathrm{kg}$ feed/week & $1-3 \mathrm{~g} / \mathrm{kg}$ feed & $195 / 100 \mathrm{~g}$ & $\begin{array}{l}\text { Square } \\
\text { Pharmaceuticals Ltd. }\end{array}$ \\
\hline Urocot & Erythromycin & $2-4 \mathrm{~g} / \mathrm{kg}$ feed & $1-5 \mathrm{~g} / \mathrm{kg}$ feed & $100 / 100 \mathrm{~g}$ & Opsonin Agrovet \\
\hline
\end{tabular}

Table 10. List of probiotics used in the study area.

\begin{tabular}{|c|c|c|c|c|c|}
\hline Trade name & Active ingredients & $\begin{array}{l}\text { Recommended } \\
\text { dose }\end{array}$ & Applied dose & $\begin{array}{l}\text { Price } \\
\text { (TK.) }\end{array}$ & $\begin{array}{l}\text { Source/ } \\
\text { Manufacturer }\end{array}$ \\
\hline Aqua photo & $\begin{array}{l}\text { Bacillus subtilis and } \\
\text { Rhodoseudomonas } \mathrm{sp.}\end{array}$ & $60-70 \mathrm{ml} / \mathrm{dec}$ & $50-60 \mathrm{ml} / \mathrm{dec}$ & 579/1 liter & ACI animal health \\
\hline Safegut & $\begin{array}{l}\text { Probiotics, vitamin and } \\
\text { enzyme }\end{array}$ & $1 \mathrm{~g} / \mathrm{Kg}$ feed & $1-2 \mathrm{~g} / \mathrm{kg}$ feed & $300 / 250 \mathrm{~g}$ & $\begin{array}{l}\text { SK+F } \\
\text { Pharmaceuticals Ltd. }\end{array}$ \\
\hline $\begin{array}{l}\text { Aqua magic } \\
\text { plus }\end{array}$ & $\begin{array}{l}\text { Azotabactor } \\
\text { chorococcum, Bacillus } \\
\text { subtillis, Candida utilis }\end{array}$ & $5-8 \mathrm{~kg} / \mathrm{acre}$ & $\begin{array}{l}5-6 \mathrm{~kg} / \mathrm{acre} \\
(50-60 \mathrm{~g} / \mathrm{dec})\end{array}$ & $750 / 5 \mathrm{~kg}$ & Fishtech Ltd. \\
\hline Ecorich & $\begin{array}{l}\text { Probiotics, mineral, } \\
\text { geolite }\end{array}$ & $1-1.5 \mathrm{~kg} / 33 \mathrm{dec}$ & $\begin{array}{l}1.3-1.6 \mathrm{~kg} / 33 \mathrm{dec} \\
(40-50 \mathrm{~g} / \mathrm{dec})\end{array}$ & $800 / 5 \mathrm{~kg}$ & $\begin{array}{l}\text { Opsonin } \\
\text { Agrovet }\end{array}$ \\
\hline Biomax & Probiotics and nutrients & $\begin{array}{l}4-5 \mathrm{~kg} / \mathrm{acre} / 3 \mathrm{ft} . \\
\text { water }\end{array}$ & $\begin{array}{l}5 \mathrm{~kg} / \mathrm{acre} \\
(50 \mathrm{~g} / \mathrm{dec})\end{array}$ & $410 / \mathrm{kg}$ & $\begin{array}{l}\text { Square } \\
\text { pharmaceuticals Ltd. }\end{array}$ \\
\hline Profs & $\begin{array}{l}\text { Bacillus sp. and } \\
\text { Pedicoccus sp. }\end{array}$ & $50-70 \mathrm{gm} / 33 \mathrm{dec}$ & $2-3 \mathrm{~g} / \mathrm{dec}$ & $660 / 100 \mathrm{~g}$ & $\begin{array}{l}\text { Eon Animal Health } \\
\text { Ltd. }\end{array}$ \\
\hline Pond guard & $\begin{array}{l}\text { Bacillus sp., Nitromonus } \\
\text { sp., Nitrobacter } \mathrm{sp} .\end{array}$ & $10-12 \mathrm{~kg} / \mathrm{acre}$ & $\begin{array}{l}10 \quad \mathrm{~kg} / \mathrm{acre} \\
(100 \mathrm{~g} / \mathrm{dec})\end{array}$ & $800 / 5 \mathrm{~kg}$ & $\begin{array}{l}\text { ACI Animal Health } \\
\text { Ltd. }\end{array}$ \\
\hline Pond care & Probiotics & $50 \mathrm{~g} / \mathrm{acre}$ & $50-100 \mathrm{~g} / \mathrm{acre}$ & $495 / 50 \mathrm{~g}$ & $\begin{array}{l}\text { SK+F } \\
\text { Pharmaceuticals Ltd. }\end{array}$ \\
\hline
\end{tabular}


Table 11. List of growth promoters used in the study area.

\begin{tabular}{|c|c|c|c|c|c|}
\hline Trade name & Active ingredients & $\begin{array}{l}\text { Recommended } \\
\text { dose }\end{array}$ & Applied dose & Price (TK.) & $\begin{array}{l}\text { Source/ } \\
\text { Manufacturer }\end{array}$ \\
\hline Panvit aqua & $\begin{array}{l}\text { Vit- A, } D_{3}, B_{1}, B_{2}, B_{6} \\
\text { Ascorbic acid }\end{array}$ & $5-10 \mathrm{ml} / \mathrm{kg}$ feed & $5-6 \mathrm{ml} / \mathrm{kg}$ feed & $125 / 100 \mathrm{ml}$ & $\begin{array}{l}\text { Square } \\
\text { Pharmaceuticals Ltd. }\end{array}$ \\
\hline GPA & $\begin{array}{l}\text { Multi stain probiotics, } \\
\text { enzymes }\end{array}$ & $0.5-1 \mathrm{~g} / \mathrm{kg}$ feed & $1 \mathrm{~g} / \mathrm{kg}$ feed & $1000 / 500 \mathrm{~g}$ & $\begin{array}{l}\text { Opsonin } \\
\text { Agrovet }\end{array}$ \\
\hline Nutrigel & $\begin{array}{l}\text { Vitamin, mineral, } \\
\text { probiotic }\end{array}$ & $5-10 \mathrm{ml} / \mathrm{kg}$ feed & $5-6 \mathrm{ml} / \mathrm{kg}$ feed & $650 / 1$ liter & $\begin{array}{l}\text { SK }+\mathrm{F} \\
\text { Pharmaceuticals Ltd. }\end{array}$ \\
\hline $\begin{array}{l}\text { Vitamix- } \mathrm{F} \\
\text { aqua premium }\end{array}$ & $\begin{array}{l}\text { Vitamin, minerals, } \\
\text { amino acid }\end{array}$ & $2.5 \mathrm{~kg} / \mathrm{ton}$ feed & $\begin{array}{l}1-2 \mathrm{~kg} / \mathrm{ton} \\
\text { feed }(1-2 \mathrm{~g} / \mathrm{kg})\end{array}$ & $330 / \mathrm{kg}$ & ACME Laboratories \\
\hline Spa gelly & Omega-3 fatty acid & $10-15 \mathrm{ml} / \mathrm{kg}$ feed & $\begin{array}{l}10-12 \mathrm{ml} / \mathrm{kg} \\
\text { feed }\end{array}$ & $580 / 1$ liter & $\begin{array}{l}\text { Eon Pharmaceuticals } \\
\text { Ltd. }\end{array}$ \\
\hline Charger gel & $\begin{array}{l}\text { 1-3 D glucan, } \\
\text { polysaccharides, Betain, } \\
\beta \text {-glucans }\end{array}$ & $2-4 \mathrm{~g} / \mathrm{kg}$ feed & $2-4 \mathrm{~g} / \mathrm{kg}$ feed & $1060 / \mathrm{kg}$ & Fishtech Ltd. \\
\hline Aci super-fish & $\begin{array}{l}\text { Vitamin, } \\
\text { Mineral, amino acids }\end{array}$ & $1-2.5 \mathrm{~kg} /$ ton feed & $2-3 \mathrm{~g} / \mathrm{kg}$ feed & $550 / 2.5 \mathrm{~kg}$ & $\begin{array}{l}\text { ACI Animal Health } \\
\text { Ltd. }\end{array}$ \\
\hline Rena Fish & $\begin{array}{l}\text { Vit-A, } \mathrm{B}_{1}, \mathrm{~B}_{2}, \mathrm{~B}_{6}, \mathrm{~B}_{12}, \\
\mathrm{C}, \mathrm{D}_{3}, \mathrm{E}, \mathrm{K}, \mathrm{Cu}, \mathrm{Mn}, \mathrm{Fe}, \\
\mathrm{Co}, \mathrm{I}, \mathrm{Ca}_{2} \mathrm{CO}_{3} \text { etc. }\end{array}$ & $1 \mathrm{~kg} /$ ton feed & $1-2 \mathrm{~g} / \mathrm{kg}$ feed & $300 / \mathrm{Kg}$ & $\begin{array}{l}\text { Renata } \\
\text { Pharmaceuticals Ltd. }\end{array}$ \\
\hline $\begin{array}{l}\text { Chemovit } \\
\text { Aqua }\end{array}$ & $\begin{array}{l}\text { Vit-A, B, C, D }, ~ E, ~ K, \\
\mathrm{Cu}, \mathrm{Mn}, \mathrm{Fe}, \mathrm{Co} \text { etc. }\end{array}$ & $2 \mathrm{~g} / \mathrm{kg}$ feed & $1-2 \mathrm{~g} / \mathrm{kg}$ feed & $430 / \mathrm{kg}$ & $\begin{array}{l}\text { Chemist } \\
\text { Laboratories Ltd. }\end{array}$ \\
\hline Aquazyme & $\begin{array}{l}\text { Sodium sulphate, } \\
\text { polyvinyl alcohol, } \\
\text { starch, selenium, } \\
\text { magnesium, silicate }\end{array}$ & $0.5-1 \mathrm{~g} / \mathrm{kg}$ feed & $1-2 \mathrm{~g} / \mathrm{kg}$ feed & $335 / 500 \mathrm{~g}$ & $\begin{array}{l}\text { Eon Animal Health } \\
\text { Products Ltd. }\end{array}$ \\
\hline
\end{tabular}

Table 12. Knowledge of aqua chemicals and drugs.

\begin{tabular}{lll}
\hline Source of knowledge & Number of farmers $(\mathbf{n}=\mathbf{1 0 0})$ & \% of farmers \\
\hline Company representatives & 76 & 76 \\
Discussion (chemical sellers, hatchery owners, other farmers) & 58 & 58 \\
Government Organization (GO) & 47 & 47 \\
\hline
\end{tabular}

Table 13. Problems faced by the fish farmers in the study area.

\begin{tabular}{lcc}
\hline Problems & Number of farmers $(\mathbf{n}=\mathbf{1 0 0})$ & \% \\
\hline Diseases & 88 & 88 \\
Low quality of fish seed & 63 & 63 \\
Lack of technical knowledge & 58 & 58 \\
Lack of finance & 55 & 55 \\
Low price of the end product & 46 & 46 \\
Insufficient water in dry season & 41 & 41 \\
Price of feeds and chemicals & 39 & 39 \\
Flood/overflow & 36 & 36 \\
Lack of manpower & 30 & 30 \\
Problem of pond leasing & 27 & 27 \\
Theft of fish & 21 & 21 \\
\hline
\end{tabular}

\section{Discussion}

Various types of chemicals and aqua-drugs have become a noteworthy part of successful aquaculture production (Rahman et al., 2017). From the present study, it was observed that fish farmers used different types of commercial chemicals and aqua-drugs which were broadly categorized as chemicals used for removal of unwanted species, for pond preparation and water quality management, to increase dissolve oxygen concentration, to reduce harmful gasses, as disinfectants, antibiotics, growth promoters and probiotics against diseases. 
The present study revealed that Lime, Potassium permanganate, Salt, Phitkari, Copper sulphate, Urea and TSP were commonly used by the selected fish farmers as traditional chemicals. These are used for pond preparation, to increase primary productivity, maintain good water quality which is more likely to the findings of Uddin $e t$ al. (2017) in Sylhet district and Ali (2008). To eradicate undesirable species farmers of Rangpur region used various chemicals such as Sumithion, Engreb, I-mec, Sanmarine, Rota plus, Tea seed meal and Nigotox aqua which is more or less similar to the findings of Anwar et al. (2018) and Rahman et al. (2015) In the study area Aquapure, Biopond, Zeofresh, JV-zeolite, Zeorich, Geo-prime, Megazeo plus, Zeolite gold, Geo-ren and Acme zeolite were used for pond preparation and water quality management by the selected fish farmers which is analogous to the study of Rahman et al. (2015).

Typically, successful fish culture depends on careful management of oxygen concentration in the culture ponds. In the current study Oxymax, Oxymore, Oxylife, Aci-ox, Oxyrich Tab, Oxygold, Oxy-aqua, Oxy-A, Oxyflox and Oxyren were used by the selected fish farmers to increase oxygen concentration. In line with the present study, earlier research findings revealed that ACI-OX $28 \%$, oxymax $22 \%$, oxy more $11 \%$, oxy flow $22 \%$ were used for oxygenation into water in Comilla region (Rahman et al., 2017); Oxyflow, Oxymax, Bio-Ox, Oxy-A and Oxy Gold were used to increase dissolved oxygen in aquaculture ponds (Monsur, 2012). Selected fish farmers in Rangpur region used Gasonil, Gastrap, Bio aqua-50, Gasonex, Metrix, Yucca gold,Pondkleen, Ammo check and Yuka etc. to remove harmful gases. And the findings can be correlated with the previous study done in the north-eastern region in Bangladesh by Rahman et al. (2015).

In the study area, diverse types of fish diseases such as tail and fin rot, Epizootic Ulcerative Syndrome (EUS), dropsy, exophthalmia, gill rot, argulosis and Chhanel Catfish Virus Disease (CCVD) were observed. Similar conditions were also reported by the various authors in previous studies in aquaculture of Bangladesh (DoF, 2002; Faruk et al., 2004). Farmers of the investigated areas used several chemicals as disinfectants to maintain hygiene and in some cases to prevent/treat a wide variety of viral, bacterial and fungal infection. Timsen, Virex, Polgard plus, Bleaching powder,Aquakleen, Pond Safe, Biokleen Aqua, Sansure and Micronil were used by the farmers of the study area as disinfectants which is comparable to the findings of Anwar et al. (2018) in Jamalpur sadar upazilla of Bangladesh. In Rangpur region various types of antibiotics are used for disease treatment by the fish farmers like Renamycin, Eskamycin, Bactitab, Cotrim vet bolus, Chemycin, Aquamycin, Oxy-D Vet, Oxin WS, Otetra vet power-50 and Urocot. The active ingredients of such antibiotics were mainly oxytetracyclin, chlortetracycline, erythromycin, sulphamethoxazole, trimethoprim. It was noticed that antibiotics were used extensively without ensuing the recommended doses. Rahman et al. (2017) mentioned that oxytetracycline $44 \%$, cotrim-vet $17 \%$, amoxicillin $28 \%$ and chlortetracycline $11 \%$ were used as antibiotics for disease treatment in Comilla regions which is more or less similar to the findings of Chowdhury et al. (2012) and Sharker et al. (2014).

In the study area different types of probiotics including Pond care, Profs, Biomax, Aqua photo, Safegut, Aqua Magic plus, Ecorich and Pond guard were used by the selected fish farmers which is well supported by Rahman et al. (2017) in Comilla region and Alam and Rashid (2014) in Shatkhira district. Commercial farmers have a tendency to get their end product as soon as possible. Aquazyme, Panvit aqua, Nutrigel, Charger gel, GPA, Vitamix- F aqua premium, Spa gelly, Aci super-fish, Rena fish and Chemovit Aqua were applied by the selected fish farmers in Rangpur district as growth promoters as in Jamalpur sadar upazilla (Anwar et al., 2018) and Shatkhira district (Alam and Rashid, 2014).

There is no doubt that chemicals are useful. It was found that most of the farmers used chemicals indiscriminately without knowing their mode of action, doses and appropriate procedures of application. Aqua drugs should not be used, if these are hazardous to the surrounding environment. Pharmaceutical companies should conduct more research works to reduce the harmful effect of aqua medicines on aquaculture.

\section{Conclusions}

After completion of the present investigation it is somehow clear that the farmers of Rangpur region are using various chemicals and aqua drugs sometimes without having proper knowledge about the doses and method of application. Unfortunately, little care has been paid on the documentation of those aqua-medicines. As a result, there is a lack of information regarding the impact of those aqua-medicines. Basically, appropriate chemicals with proper doses can minimize the adverse effects of those chemicals in aquaculture but negligence can become a threat for the future.

\section{Acknowledgements}

The authors would like to express their sincere appreciation to Institute of Research and Training (IRT) for funding this research. 


\section{Conflict of interest}

None to declare.

\section{References}

Alam MA and MM Rashid, 2014. Use of aqua-medicines and chemicals in aquaculture in Shatkhira District, Bangladesh. J. Pharm. Biol. Sci., 9: 5-9.

Ali MM, 2008. Study on the chemicals and antibiotics used in aquatic animal health management. An MS Thesis, Department of Aquaculture, Bangladesh Agricultural University, Mymensingh. pp. 1-54.

Anwar MA, MM Rashid, MAHM Kamal, MM Rahman and D Pandit, 2018. Aqua Drugs and Chemicals Used in Aquaculture in Jamalpur Sadar Upazila of Bangladesh. Asian J. Fish. Aquat. Res., 2: 1-13.

BFRI, 1999. Fish diseases: prevention and control. Bangladesh Fisheries Research Institute, Circular leaflets no 6. 2nd edition. p. 7.

Chowdhury AJ, D Saha, MB Hossain, M Shamsuddin and MH Minar, 2012. Chemicals used in freshwater aquaculture with special emphasis to fish health management of Noakhali, Bangladesh. African J. Basic App. Sci., 4: 110-114.

DoF, 2002. Fish Fortnight Compendium. 10-24 August 2002, Department of Fisheries, Matsha Bhaban, Dhaka. pp. 44-45.

Faruk MAR, MJ Alam, MMR Sarker and MB Kabir, 2004. Status of fish disease and health management practices in rural freshwater aquaculture of Bangladesh. Pak. J. Biol. Sci., 7: 2092-2098.

Hossain MK, MS Haq, BK Chawkraborty, MT Hasan and SK Mazumder, 2014. Present status of aquamedicines used for fish culture at shantahar and Adamdighi of Bogra district, Bangladesh. IOSR J. Environ. Sci. Toxic. Food Technol., 8: 37-42.

Karim M and J Stellwagen, 1998. Final Report on Fourth Fisheries Projects: shrimp aquaculture (Preparatory phase for National Development Program). Department of Fisheries, Ministry of Fisheries \& Livestock, Bangladesh.

Monsur A, 2012. Use of aqua drugs and chemicals of aquaculture in Jamalpur and Sherpur region. MS Thesis, Department of Aquaculture, Bangladesh Agricultural University, Mymensingh.

Rahman MM, MMM Alam, SMI Khalil, SM Bari and MM Rashid, 2015. Status of chemicals and aqua drugs used in freshwater aquaculture in north-eastern Bangladesh. J. Sylhet Agril. Univ., 2: 243-252.

Rahman MZ, A Khatun, MI Kholil and MMM Hossain, 2017. Aqua drugs and chemicals used in fish farms of Comilla regions. J. Entomol. Zool. Stud., 5: 2462-2473.

Shamsuzzaman MM and TK Biswas, 2012. Aqua chemicals in shrimp farm: A study from south-west coast of Bangladesh. Egypt. J. Aquat. Res., 38: 275- 285.

Sharker MR, KR Sumi, MJ Alam, MM Rahman, Z Ferdous, MM Ali and MR Chaklader, 2014. Drugs and chemicals used in aquaculture activities for fish health management in the coastal region of Bangladesh. Int. J. Life Sci. Biotech. Pharma Res., 3: 49-58.

Subasinghe RP, U Barg and A Tacon, 1996. Chemicals in Asian Aquaculture: need, usage, issues and challenges. In: Use of Chemicals in Aquaculture in Asia, Arthur JR, CR Lavilla-Pitogo, RP Subasinghe (Editors). Southeast Asian Fisheries Development Center, Aquaculture Development Tigbauan, lloilo, Philippines. pp. 1-5.

Uddin S, MA Hossain, S Ahamed, MM Iqbal and M Akter, 2017. Status of drugs, chemicals and antibiotics usages in freshwater aquaculture activities at Jaintapurupazila of Sylhet, Bangladesh. Algerian J. Environ. Sci. Technol., 3: 368-373. 